\title{
Clinical value of serum Ape1/Ref-1 combined with TGF- $\beta 1$ monitoring in predicting the occurrence of radiation pneumonitis $(R P)$ in non-small cell lung cancer patients
}

\author{
Yan Zhang ${ }^{1,2 \#}$, Hongjun You ${ }^{2,3 \#}$, Jun Duan ${ }^{1,2}$, Yang Gao ${ }^{2,4}$ \\ ${ }^{1}$ Department of Geriatric Medicine, Sichuan Provincial People's Hospital, University of Electronic Science and Technology of China, Chengdu, \\ China; ${ }^{2}$ Chinese Academy of Sciences, Sichuan Translational Medicine Research Hospital, Chengdu, China; ${ }^{3}$ Department of Respiratory and Critical \\ Illness, Sichuan Provincial People's Hospital, University of Electronic Science and Technology of China, Chengdu, China; ${ }^{4}$ Department of General \\ Practice Center, Sichuan Provincial People's Hospital, University of Electronic Science and Technology of China, Chengdu, China \\ Contributions: (I) Conception and design: Y Zhang, H You; (II) Administrative support: J Duan, Y Gao; (III) Provision of study materials or patients: \\ Y Zhang, H You; (IV) Collection and assembly of data: Y Zhang, H You; (V) Data analysis and interpretation: Y Zhang, H You; (VI) Manuscript \\ writing: All authors; (VII) Final approval of manuscript: All authors. \\ "These authors contributed equally to this work. \\ Correspondence to: Jun Duan. Department of Geriatric Medicine, Sichuan Provincial People's Hospital, University of Electronic Science and \\ Technology of China, No. 32 W. Sec 2, 1st Ring Road, Qingyang District, Chengdu, China. Email: qzlxnjlough016@sina.com; Yang Gao. \\ Department of General Practice Center, Sichuan Provincial People's Hospital, University of Electronic Science and Technology of China, No. 32 W. \\ Sec 2, 1st Ring Road, Qingyang District, Chengdu, China. Email: 63309674@qq.com.
}

Background: The aim of the present study was to explore the predictive value of serum apurinic/apyrimidinic endonuclease 1/redox factor-1 (Ape1/Ref-1) combined with transforming growth factor $\beta 1$ (TGF- $\beta 1$ ) levels in the occurrence of radiation pneumonitis (RP) in patients with non-small cell lung cancer (NSCLS).

Methods: Eighty-one patients with NSCLS who were admitted from August 2017 to July 2019 were enrolled in the present study. All patients were treated with concurrent radiotherapy and chemotherapy. Serum Ape1/Ref-1 and TGF- $\beta 1$ levels were measured before treatment and 12 weeks after treatment. Patients with radiation-induced lung injury were assessed and divided into the RP group (lung injury $\geq 2$ ) and non-RP (NRP) group (grade <2). The levels of serum Ape1/Ref-1 and TGF- $\beta 1$ before and after treatment between the 2 groups were compared. The relationship between clinical characteristics, serum Ape1/Ref1 , TGF- $\beta 1$ levels, and the occurrence of RP were then analyzed, and the relationship between serum Ape1/ Ref-1, TGF- $\beta 1$ levels, and their predictive value for the occurrence of RP was also assessed.

Results: The incidence of RP in 81 patients was 30.86\%. After treatment, the serum Ape1/Ref-1 and TGF- $\beta 1$ levels of the 2 groups were significantly higher than those before treatment $(\mathrm{P}<0.05)$. Furthermore, after treatment, the levels of serum Ape1/Ref-1 and TGF- $\beta 1$ in the RP group were significantly higher than those of the NRP group $(\mathrm{P}<0.05)$. Multivariate logistic regression analysis showed that V20, Ape1/Ref1 , and TGF- $\beta 1$ were associated with the occurrence of $\mathrm{RP}(\mathrm{P}<0.05)$. The levels of serum Ape1/Ref-1 were positively correlated with TGF- $\beta 1(r=0.734, \mathrm{P}<0.05)$. Finally, the area under the curve of RP occurrence, which was predicted by the levels of serum Ape1/Ref-1, TGF- $\beta 1$, and the combination of both were 0.779 , 0.69 , and 0.842 , respectively.

Conclusions: The occurrence of RP in NSCLS patients is closely related to the levels of serum Ape1/Ref1 and TGF- $\beta 1$, and the combination of both has important predictive values for the occurrence of RP.

Keywords: Apurinic/apyrimidinic endonuclease 1/redox factor-1 (Ape1/Ref-1); transforming growth factor $\beta 1$ (TGF- $\beta 1$ ); non-small cell lung cancer (NSCLS); radiation pneumonitis (RP); predictive value

Submitted Jan 27, 2021. Accepted for publication Mar 23, 2021.

doi: $10.21037 /$ apm-21-423

View this article at: http://dx.doi.org/10.21037/apm-21-423 


\section{Introduction}

Lung cancer is the most common cancer in incidence and mortality worldwide. There is a 1.8 million increase in the number of lung cancer patients worldwide each year, and $30 \%$ of patients with malignant tumors die of lung cancer (1). Non-small cell lung cancer (NSCLS) is a common type of lung cancer, and the prognosis of patients is relatively poor. The current standard treatment for NSCLS is concurrent radiotherapy and chemotherapy (2). Although concurrent radiotherapy and chemotherapy can effectively improve the prognosis of NSCLS patients, patients are also prone to various complications; radiation pneumonitis (RP) is an important complication associated with its treatment. Studies have shown that after the occurrence of RP, alveolar endothelial cells and epithelial cells are frequently injured, followed by fibroblast proliferation, fibrous matrix formation, and abnormal changes in the levels of inflammatory factors. Therefore, the occurrence of $\mathrm{RP}$ is closely related to the levels of cytokines related to the above changes (3). As a multifunctional protein, apurinic/ apyrimidinic endonuclease 1/redox factor-1 (Ape1/Ref-1) plays an important role in the occurrence of RP, and oxidative stress caused by radiotherapy can increase the level of Ape1/Ref-1 (4). The research report of Yu et al. (5) pointed out that ionizing radiation can stimulate the overexpression of APE1/Ref-1 protein, and can cause it to shift from the nucleus to the cytoplasm and the expression of the first increase and then decrease, indicating that Ape1/Ref-1 can be used as a molecular target for the prevention and treatment of radiation-induced lung injury, and may play an important role in damage repair during the occurrence and development of radiation-induced lung injury. Transforming growth factor $\beta 1$ (TGF- $\beta 1$ ) is related to the process of pulmonary fibrosis. It can regulate cellmatrix proteins by activating fibroblasts, thereby affecting collagen synthesis, and mediate RP occurrence and its repair processes (6). According to the research, TGF- $\beta 1$ can positively express in the sputum of patients with NSCLC, located in macrophages and epithelial cells, with macrophages as the main areas of expression. Patients with positively expressed TGF- $\beta 1$ in sputum after radiotherapy have a higher incidence of radiation pneumonitis than those with negative expressions (7). Studies have shown that the detection of serum Ape1/Ref-1, ICAM-1 and IL-17A levels in 68 eligible advanced NSCLC patients with concurrent radiotherapy and chemotherapy before and after treatment has found that the serum IL-17A may be used to predict the occurrence of radiation pneumonitis. However, the occurrence of radiation-induced lung injury is a complex process involving multiple cytokines, and changes in a single cytokine may not accurately reflect this process (8). In the present study, we confirmed that Ape1/Ref- 1 and TGF- $\beta 1$ are related to the occurrence of $\mathrm{RP}$, but whether they can be used individually or concurrently to predict the occurrence of RP needs further research. Therefore, we explored the relationship between serum Ape1/Ref-1, TGF- $\beta 1$ levels, and the occurrence of RP in NSCLS patients. We present the following article in accordance with the STARD reporting checklist (available at http://dx.doi.org/10.21037/ apm-21-423).

\section{Methods}

\section{General information}

Eighty-one patients with NSCLS who were admitted from August 2017 to July 2019 were enrolled in the present study. All patients agreed to participate in this study and signed an informed consent form. The study was conducted in accordance with the Declaration of Helsinki (as revised in 2013). This study was approved by the Sichuan Provincial People's Hospital, University of Electronic Science and Technology of China (No. 20170626). The inclusion criteria were as follows: (I) NSCLS patients with no distant metastasis; (II) stages I-III tumors according to the American Joint Committee on Cancer staging (9); (III) patients had not received antitumor therapy prior to enrollment; and (IV) patients received and met the indications of concurrent radiotherapy and chemotherapy. Exclusion criteria were as follows: (I) other tumors; (II) abnormal mental; (III) contraindications to concurrent radiotherapy and chemotherapy; (IV) pulmonary fibrosis or pulmonary infectious diseases before treatment; (V) previous lung resection. Of the 81 patients, 39 were males and 42 were females. Their ages ranged from 39 to 83 years, with an average age of $61.29 \pm 10.29$ years. Based on American Joint Committee on Cancer staging, 23 cases were stage I, 28 cases were stage II, and 30 cases were stage III. The course of illness was $1-56$ months, with an average of $39.22 \pm 10.21$ months.

\section{Treatment plan}

The patients were treated with reverse intensity-modulated chemotherapy. After the vacuum body frame or the heat shrinkable body film was fixed, computed tomography 
(CT) simulation positioning scan was used, and the interval between the layers was $3 \mathrm{~mm}$. The Varian EclipseDX system (Palo Alto, CA, USA) was then used to delineate the treatment target area. The general target area was divided according to the visible lesions in the imaging examination, whereas the clinical target area was expanded outside the general target area, of which squamous cell carcinoma and adenocarcinoma were expanded by 6 and 8 $\mathrm{mm}$, respectively. The dose-volume histogram and isodose curve were used to determine the radiotherapy treatment plan in consideration of the patient's lung function and its involvement. The dose of the radiotherapy was from 60 to $70 \mathrm{~Gy}$, with a total of 30 to 35 times, spanning 6-7 weeks. The maximum, minimum, and average doses in the general target area, lung V5, V10, V20, and average dose, and normal tissue complication rate were collected to ensure that normal tissues were not affected by radiotherapy. The concurrent chemotherapy included 2 cycles. Specifically, the treatment included cisplatin combined with etoposide. Cisplatin was used at $50 \mathrm{mg} / \mathrm{m}^{2}$ on the $1 \mathrm{st}, 8 \mathrm{th}, 29 \mathrm{th}$, and 36th day of treatment, whereas $50 \mathrm{mg} / \mathrm{m}^{2}$ etoposide was applied on days $1-5$ and $29-33$ of treatment. Following treatment, the patients were followed-up every 3 months.

\section{Determination of serum index}

A total of $3 \mathrm{~mL}$ of fasting venous blood in the morning after the treatment was completed and before the treatment of the patient was collected, and the serum was then obtained after centrifugation. Enzyme-linked immunosorbent assay was then used to determine the levels of Ape1/Ref- 1 and TGF- $\beta 1$. The determination kit was purchased from R\&D Systems (USA). The determination step was performed according to the manufacturer's instructions.

\section{Determination of RP}

The RP grade was determined based on the relevant physical signs and chest $\mathrm{CT}$ imaging examination results, according to the standards issued by the European Treatment Cooperative Group and the American Oncology Radiotherapy Cooperative Group (9). Grade 0 means that there are no significant changes in related signs compared with those of pre-treatment; grade 1 means that there is a mild cough and shortness of breath when coughing hard after treatment; grade 2 means that after treatment there are continuous coughing symptoms, the patient needs medication for antitussive, and breathing difficulties occur when exerting force; this symptom disappears in the resting state; grade 3 means that after treatment there is symptomatic severe cough and the application of antitussive drugs is ineffective; symptoms of shortness of breath even appear in the resting state, and the imaging examination shows acute pneumonia, so patients may require corticosteroids or intermittent oxygen therapy; grade 4 means that after treatment there is severe respiratory insufficiency requiring assisted ventilation therapy or continuous oxygen inhalation. Patients with grade $<2$ were defined as RP, and all patients were then divided into the RP group and non-RP (NRP) group based on the classification results.

\section{Analysis of observation indicators}

The incidence of RP in patients was analyzed, and the levels of serum Ape1/Ref-1, TGF- $\beta 1$ before and after treatment between the 2 groups were then compared. After that, the relationship between clinical features, serum Ape1/Ref-1, TGF- $\beta 1$ levels, and the occurrence of RP were assessed. Finally, the correlation between serum Ape1/Ref-1 and TGF- $\beta 1$ levels was analyzed, and the predictive value of Ape1/Ref- 1 and TGF- $\beta 1$ levels for the occurrence of RP were explored.

\section{Statistical analysis}

SPSS version 20.0 (Armonk, NY, USA) software was used to process the data generated in the present study. In particular, measurement data, such as serum index levels, were expressed as mean \pm standard deviation, $t$-test was used for comparison, count data were expressed as the percentage, and $\chi^{2}$-test was used for the comparison of differences. The relationship between clinical characteristics, serum Ape1/Ref-1, TGF- $\beta 1$ levels, and the occurrence of RP were analyzed by single-factor and multiple logistic regression analysis. Pearson correlation analysis was used, and the receiver-operating characteristic (ROC) curve was used to measure the predictive value of each variable for the occurrence of RP. $\mathrm{P}<0.05$ indicated that the difference was statistically significant.

\section{Results}

\section{Analysis of RP incidence}

Patients were followed-up until October 2020. The followup time was 15-26 months, the median follow-up time was 
Table 1 Comparison of serum apurinic/apyrimidinic endonuclease 1/redox factor-1 (Ape1/Ref-1) and transforming growth factor $\beta 1$ (TGF- $\beta 1$ ) levels before and after treatment in the 2 groups

\begin{tabular}{lcccr}
\hline Groups & Cases & Time & Ape1/Ref-1 (ng/mL) & TGF- $\beta 1(\mathrm{ng} / \mathrm{mL})$ \\
\hline RP group & 25 & Before treatment & $3.01 \pm 0.91$ & $7.52 \pm 2.94$ \\
& & After treatment & $4.96 \pm 1.15$ & $14.29 \pm 3.41$ \\
NRP group & 56 & Before treatment & $3.15 \pm 0.96$ & $7.76 \pm 3.06$ \\
& & After treatment & $3.86 \pm 1.27$ & $10.51 \pm 3.14$ \\
\hline
\end{tabular}

NRP, non-radiation pneumonitis; RP, radiation pneumonitis.

23 months, and no one was excluded from follow-up. Of the 81 patients, 25 had RP, and the incidence of RP was $30.86 \%$. Fourteen cases were grade 2, 10 cases were grade 3, and 1 case was grade 4 .

\section{Comparison of serum Ape1/Ref-1 and TGF-p1 levels before and after treatment in the 2 groups}

The serum Ape1/Ref- 1 and TGF- $\beta 1$ levels before treatment between the 2 groups were not statistically significant $(\mathrm{P}>0.05)$. After treatment, the serum Ape1/Ref-1 and TGF- $\beta 1$ levels of the 2 groups both significantly increased compared with before treatment $(\mathrm{P}<0.05)$. Furthermore, after treatment, the serum Ape1/Ref- 1 and TGF- $\beta 1$ levels in the RP group were significantly higher than those in the NRP group $(\mathrm{P}<0.05)$, as shown in Table 1.

\section{Relationship between patients' clinical characteristics and the occurrence of $R P$}

Radiotherapy dose and V20 were related to the occurrence of $\mathrm{RP}(\mathrm{P}<0.05)$, whereas sex, age, pathological type, tumor location, smoking history, and clinical stage were not related to the occurrence of RP $(\mathrm{P}>0.05)$, as shown in Table 2.

\section{Multivariate analysis of RP occurrence}

The results of the multiple regression analysis showed that V20, Ape1/Ref- 1 , and TGF- $\beta 1$ were independent influencing factors of RP occurrence $(\mathrm{P}<0.05)$, whereas radiotherapy dose was not an independent influencing factor of RP occurrence $(\mathrm{P}>0.05)$, as shown in Table 3.

\section{Correlation analysis between serum Ape1/Ref-1 and TGF-ß1 levels}

Pearson correlation analysis showed that serum Ape1/
Ref-1 levels were positively correlated with TGF- $\beta 1$ levels $(\mathrm{r}=0.734, \mathrm{P}<0.05)$.

\section{Serum Ape1/Ref-1, TGF-ß1 levels, and the combination of both for the predictive value of RP occurrence}

ROC curve results showed that the area under the curve (AUC) for RP occurrence predicted by serum Ape1/Ref1 and TGF- $\beta 1$ was 0.779 and 0.694 , respectively, and the AUC predicted by the combination of both was 0.842 , as shown in Figure 1 and Table 4.

\section{Discussion}

$\mathrm{RP}$ is an important complication affected by radiation dose during radiotherapy of lung cancer. Its incidence during the treatment of patients is in the range of $13-37 \%$ (10). The incidence of RP in the present study was $30.86 \%$, which was within the reported range. In the treatment of radiation pneumonia, in addition to symptomatic treatment such as relieving cough and expectorating phlegm, the treatment of glucocorticoid is the key. It is recommended to use longacting dexamethasone or prednisone, starting with a smaller individualized dose, adjusting to an effective dose according to the condition and maintaining it for 3 to 4 weeks, and slowly reducing the hormone to avoid recurrence of the disease (11). The occurrence of RP is related to lung injury caused by ionizing radiation. After lung injury, inflammatory mediators cause the intense body's immune response, and the prediction of RP is beneficial to the formulation of patient treatment plans $(12,13)$. Therefore, the detection of changes in cytokine levels during the process of RP and its application in predicting the occurrence of RP have become the focus of research in the field of NSCLS.

Ape1/Ref-1 is composed of 2 functionally different subunits, Ape1 and Ref-1, which can form a highly conserved functional complex after integration. It is 
Table 2 Analysis of the relationship between patients' clinical characteristics and the occurrence of radiation pneumonitis (RP)

\begin{tabular}{|c|c|c|c|c|}
\hline Characteristics & RP group (n=25), n (\%) & NRP group (n=56), n (\%) & $\chi^{2}$ value & $P$ value \\
\hline Male & $10(40.00)$ & $29(60.00)$ & & \\
\hline Female & $15(60.00)$ & $27(48.21)$ & & \\
\hline Age (years) & & & 0.551 & 0.489 \\
\hline Pathological type & & & 0.304 & 0.861 \\
\hline Squamous cell carcinoma & $13(52.00)$ & $26(48.15)$ & & \\
\hline Adenocarcinoma & $9(36.00)$ & $19(35.19)$ & & \\
\hline Other & $3(12.00)$ & $9(16.70)$ & & \\
\hline Lower left & $7(28.00)$ & $14(25.00)$ & & \\
\hline Upper right & $5(20.00)$ & $12(21.43)$ & & \\
\hline Lower right & $3(12.00)$ & $8(14.29)$ & & \\
\hline Middle right & $1(4.00)$ & $5(8.93)$ & & \\
\hline Smoking history & & & 0.472 & 0.492 \\
\hline Yes & $10(40.00)$ & $18(32.14)$ & & \\
\hline No & $15(60.00)$ & $38(67.86)$ & & \\
\hline Clinical stage & & & 0.623 & 0.723 \\
\hline$\geq 60$ & $20(80.00)$ & $20(35.71)$ & & \\
\hline V20 (\%) & & & 4.241 & 0.040 \\
\hline$<17.3$ & $9(36.00)$ & $34(60.71)$ & & \\
\hline$\geq 17.3$ & $16(64.00)$ & 22 (39.29) & & \\
\hline
\end{tabular}

NRP, non-radiation pneumonitis; $\chi^{2}$-test, Chi-square test; $P$ value, probability; $n$, number.

Table 3 Multivariate analysis of radiation pneumonitis occurrence

\begin{tabular}{lcccccc}
\hline Factors & $\beta$ & SE & Wald $\chi^{2}$ & OR & $95 \%$ Cl & P value \\
\hline Radiation dose & 0.516 & 0.338 & 2.331 & 1.675 & $0.516-3.249$ & 0.128 \\
V20 & 0.418 & 0.196 & 4.548 & 1.519 & $0.418-2.230$ & 0.034 \\
Ape1/Ref-1 & 0.643 & 0.323 & 3.963 & 1.902 & $0.643-3.583$ & 0.047 \\
TGF- $\beta 1$ & 0.229 & 0.106 & 4.667 & 1.257 & $1.021-1.548$ & 0.031 \\
\hline
\end{tabular}

Ape1/Ref-1, apurinic/apyrimidinic endonuclease 1/redox factor-1; $\mathrm{Cl}$, confidence interval; OR, odds ratio; TGF- $\beta 1$, transforming growth factor $\beta 1$; SE, standard error; OR, odds ratio; Cl, confidence interval; P value, probability. 
involved in the occurrence and progression of a variety of malignant tumors $(14,15)$. Ionizing radiation can cause lung tissue cells to be in a state of intense oxidative stress and promote the compensatory synthesis of Ape1/Ref-1, thereby regulating the level of cellular oxidative stress transcription factors $(16,17)$. A previous study suggested that ionizing radiation can cause the overexpression of Ape1/Ref- 1 , which can gradually move from the nucleus to the cytoplasm, and the characteristics of Ape1/Ref-1 expression shows first rising and then falling, and Ape1/Ref- 1 expression plays an important role in the occurrence and progression of RP $(18,19)$. In the present study, we found that the level of serum Ape1/Ref-1 in the RP group was significantly higher than that in the NRP group, further confirming that Ape1/Ref-1 is related to the occurrence of RP. We believe that ionizing radiation can increase the formation of various reactive oxygen species in the body, which puts the body in a state of oxidative stress, and Ape1/Ref- 1 can

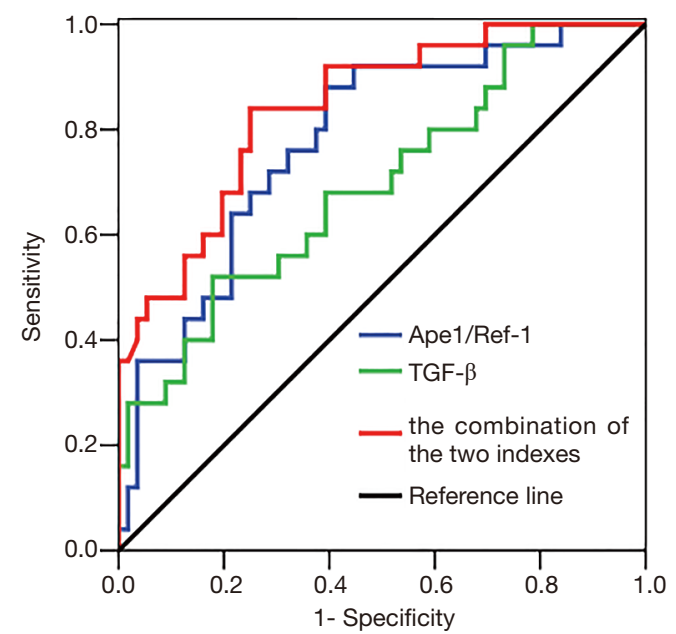

Figure 1 Receiver-operating characteristic curve of serum apurinic/apyrimidinic endonuclease 1/redox factor-1 (Ape1/Ref-1), transforming growth factor $\beta 1$ (TGF- $\beta 1$ ), and the combination of both for the prediction of radiation pneumonitis occurrence. promote the activity of 3 'diesterase through the formation of polymerase 2-hydroxy primers, which in turn reduces the damage of RP caused by reactive oxygen species, so Ape1/ Ref-1 expression level increases after RP occurs (20). In their study, Guo et al. showed that there was little difference in serum Ape1/Ref-1 levels between the RP group and the NRP group (8). They found that, although Ape1/Ref-1 is related to the occurrence of lung cancer and RP, further research was required on whether it can be a predictor of the occurrence of RP. The occurrence of RP is caused by a variety of cytokines that participate in complex pathological processes. Various damage can occur to the target cells, such as alveolar endothelial cells and type II epithelial cells, due to the influence of radiation, which then releases a variety of cytokines to induce various inflammatory reactions, leading to the activation of macrophages and release of TGF- $\beta 1$. This further accelerates the transformation of fibroblasts, promotes fibroblast proliferation, accelerates the synthesis of the matrix protein, and ultimately leads to pulmonary interstitial fibrosis (21). The findings of the present study indicated that the serum TGF- $\beta 1$ level in the RP group was significantly higher than that in the NRP group. TGF- $\beta 1$ plays an important role in the occurrence of RP. It can induce the expression of the connective tissue growth factor by mediating collagen transcription and translation, and can also mediate the phosphorylation process of $\operatorname{Smad} 2 / 3$ and promote the repair of lung injury caused by radiation (19). Animal experiments have shown the occurrence of neovascularization when RP occurs, and TGF- $\beta 1$ can regulate the level of vascular endothelial growth factor, promote the formation of reactive oxygen species, and regulate oxidative stress in the lungs. The treatment of $\mathrm{RP}$ mice with Cistanche acid can effectively reduce the inflammation and oxidative stress in the lungs, indicating that the occurrence of RP is mainly related to the activation of the TGF- $\beta 1 /$ vascular endothelial growth factor signaling pathway (22). Studies have shown that Ape1/Ref-1 is an important regulator of inflammatory indicators, which

Table 4 Analysis of serum apurinic/apyrimidinic endonuclease 1/redox factor-1 (Ape1/Ref-1), transforming growth factor $\beta 1$ (TGF- $\beta 1$ ), and the combination of both in predicting the occurrence of radiation pneumonitis

\begin{tabular}{lcccccc}
\hline Indicators & Cut-off value & AUC & Sensitivity & Specificity & $95 \% \mathrm{Cl}$ & $\mathrm{P}$ value \\
\hline Ape1/Ref-1 & $4.36 \mathrm{ng} / \mathrm{mL}$ & 0.779 & 79.6 & 82.5 & $0.673-0.885$ & $<0.001$ \\
TGF- $\beta 1$ & $12.86 \mathrm{ng} / \mathrm{mL}$ & 0.694 & 77.5 & 72.4 & $0.569-0.820$ & 0.005 \\
Combined indicators & - & 0.842 & 86.5 & 80.2 & $0.753-0.932$ & $<0.001$ \\
\hline
\end{tabular}

AUC, area under the curve; $\mathrm{Cl}$, confidence interval; $\mathrm{P}$ value, probability. 
regulates the level of inflammatory factors in the body through multiple transcription factors, such as activator protein 1 (AP-1), nuclear transcription factor-kappa B (NF$\mathrm{kB}$ ), and amino-terminal fragment (ATF) (23). However, the correlation analysis in the present study showed that there is a positive correlation between Ape1/Ref- 1 and TGF- $\beta 1$, indicating that Ape1/Ref-1 may regulate the level of body inflammatory factors, such as TGF- $\beta 1$, through a certain transcription factor, but its specific mechanism of action still needs further research. Finally, the ROC curve showed that the AUC of serum Ape1/Ref-1, TGF- $\beta 1$, and the combination of both for the prediction of the occurrence of $\mathrm{RP}$ was $0.779,0.694$, and 0.842 , respectively. The combined use of these indicators to predict the occurrence of $\mathrm{RP}$ is better than a single indicator for the prediction of the occurrence of RP.

In conclusion, the occurrence of RP in NSCLS patients is related to abnormalities in Ape1/Ref- 1 and TGF- $\beta 1$ levels. Ape1/Ref- 1 may mediate changes in TGF- $\beta 1$ levels through the regulation of related transcription factors, and the combination of Ape1/Ref- 1 and TGF- $\beta 1$ has a superior predictive value for $\mathrm{RP}$ occurrence.

\section{Acknowledgments}

Funding: None.

\section{Footnote}

Reporting Checklist: The authors have completed the STARD reporting checklist. Available at http://dx.doi.org/10.21037/ apm-21-423

Data Sharing Statement: Available at http://dx.doi. org/10.21037/apm-21-423

Conflicts of Interest: All authors have completed the ICMJE uniform disclosure form (available at http://dx.doi. org/10.21037/apm-21-423). The authors have no conflicts of interest to declare.

Ethical Statement: The authors are accountable for all aspects of the work in ensuring that questions related to the accuracy or integrity of any part of the work are appropriately investigated and resolved. The study was conducted in accordance with the Declaration of Helsinki (as revised in 2013). This study was approved by the Sichuan Provincial People's Hospital, University of Electronic
Science and Technology of China (No. 20170626). Written informed consent was obtained for publication of this study.

Open Access Statement: This is an Open Access article distributed in accordance with the Creative Commons Attribution-NonCommercial-NoDerivs 4.0 International License (CC BY-NC-ND 4.0), which permits the noncommercial replication and distribution of the article with the strict proviso that no changes or edits are made and the original work is properly cited (including links to both the formal publication through the relevant DOI and the license). See: https://creativecommons.org/licenses/by-nc-nd/4.0/.

\section{References}

1. Romaszko AM, Doboszyńska A. Multiple primary lung cancer: A literature review. Adv Clin Exp Med 2018;27:725-30.

2. Zhou W, Liu T, Saren G, et al. Comprehensive analysis of differentially expressed long non-coding RNAs in nonsmall cell lung cancer. Oncol Lett 2019;18:1145-56.

3. Hanania AN, Mainwaring W, Ghebre YT, et al. RadiationInduced Lung Injury: Assessment and Management. Chest 2019;156:150-62.

4. Fishel ML, Xia H, McGeown J, et al. Antitumor Activity and Mechanistic Characterization of APE1/ Ref-1 Inhibitors in Bladder Cancer. Mol Cancer Ther2019;18:1947-60.

5. Yu SL, Wang D, Lin L, et al. The characteristics of APE1/ Ref- 1 expression in the formation of radiation-induced lung injury in mice. Chongqing Yi Xue 2009;38:2301-3.

6. Khaleva E, Gridneva Z, Geddes DT, et al. Transforming growth factor beta in human milk and allergic outcomes in children: A systematic review. Clin Exp Allergy 2019;49:1201-13.

7. Wang J, Qiao XY, Lu FH, et al. TGF-beta1 in serum and induced sputum for predicting radiation pneumonitis in patients with non-small cell lung cancer after radiotherapy. Chinese Journal of Cancer 2010;29:325-9.

8. Guo L, Ding G, Xu W, et al. Correlations between Ape1/ Ref-1, ICAM-1 and IL-17A Levels in Serum and Radiation Pneumonitis for Local Advanced Non-small Cell Lung Cancer Patients. Zhongguo Fei Ai Za Zhi 2018;21:383-8.

9. Fleming ID, Phillips JL, Menck HR. The National Cancer Data Base report on completeness of American Joint Committee on Cancer staging in United States cancer facilities. The American College of Surgeons Commission on Cancer and the American Cancer Society. Cancer 
1996;78:1498-504.

10. Sun F, Sun H, Zheng X, et al. Angiotensin-converting Enzyme Inhibitors Decrease the Incidence of Radiationinduced Pneumonitis Among Lung Cancer Patients: A Systematic Review and Meta-analysis. J Cancer 2018;9:2123-31.

11. Feng QF, Zheng ML, Zeng Q. Diagnosis and treatment of radiation pneumonia. Chinese Journal of Radiation Oncology 2021;30:7-10.

12. Yahyapour R, Amini P, Saffar H, et al. Protective Effect of Metformin, Resveratrol and Alpha-lipoic Acid on Radiation- Induced Pneumonitis and Fibrosis: A Histopathological Study. Curr Drug Res Rev 2019;11:111-7.

13. Hara R, Itami J, Komiyama T, et al. Serum levels of KL-6 for predicting the occurrence of radiation pneumonitis after stereotactic radiotherapy for lung tumors. Chest 2004;125:340-4.

14. Gào X, Schöttker B. Reduction-oxidation pathways involved in cancer development: a systematic review of literature reviews. Oncotarget 2017;8:51888-906.

15. Deng X, Zhen P, Niu X, et al. APE1 promotes proliferation and migration of cutaneous squamous cell carcinoma. J Dermatol Sci 2020;100:67-74.

16. Xie J, Li Y, Kong J, et al. Apurinic/Apyrimidinic Endonuclease 1/Redox Factor-1 Could Serve as a Potential Serological Biomarker for the Diagnosis and Prognosis of Oral Squamous Cell Carcinoma. J Oral Maxillofac Surg 2019;77:859-66.

Cite this article as: Zhang Y, You H, Duan J, Gao Y. Clinical value of serum Ape1/Ref-1 combined with TGF- $\beta 1$ monitoring in predicting the occurrence of radiation pneumonitis (RP) in non-small cell lung cancer patients. Ann Palliat Med 2021;10(3):3328-3335. doi: 10.21037/apm-21-423
17. Cocchiola R, Rubini E, Altieri F, et al. STAT3 PostTranslational Modifications Drive Cellular Signaling Pathways in Prostate Cancer Cells. Int J Mol Sci 2019;20:1815-31.

18. Yan T, Venkat P, Chopp M, et al. APX3330 Promotes Neurorestorative Effects after Stroke in Type One Diabetic Rats. Aging Dis 2018;9:453-66.

19. Bhat AA, Lu H, Soutto M, et al. Exposure of Barrett's and esophageal adenocarcinoma cells to bile acids activates EGFR-STAT3 signaling axis via induction of APE1. Oncogene 2018;37:6011-24.

20. McIlwain DW, Fishel ML, Boos A, et al. APE1/Ref1 redox-specific inhibition decreases survivin protein levels and induces cell cycle arrest in prostate cancer cells. Oncotarget2017;9:10962-77.

21. Ghavami S, Yeganeh B, Zeki AA, et al. Autophagy and the unfolded protein response promote profibrotic effects of TGF- $\beta 1$ in human lung fibroblasts. Am J Physiol Lung Cell Mol Physiol 2018;314:L493-L504.

22. Yu YX, Xiu YL, Chen X, et al. Transforming Growth Factor-beta 1 Involved in the Pathogenesis of Endometriosis through Regulating Expression of Vascular Endothelial Growth Factor under Hypoxia. Chin Med J (Engl) 2017;130:950-6.

23. Lee YR, Joo HK, Lee EO, et al. Plasma APE1/Ref-1 correlates with atherosclerotic inflammation in ApoE-/mice. Biomedicines 2020;8:366-82.

(English Language Editor: R. Scott) 